\title{
E-Loyalty Building in Competitive E-Service Market of SNS: Resources, Habit, Satisfaction and Switching Costs
}

\author{
Yong Liu ${ }^{1, *}$, Shengli Deng ${ }^{2}$, and Feng $\mathrm{Hu}^{3}$ \\ ${ }^{1}$ Chair of Marketing and Innovation, Universität Hamburg, Hamburg, Germany \\ Yong. Liu@wiso.uni-hamburg. de \\ ${ }^{2}$ Center for study of Information Resources, Wuhan University, Wuhan, China \\ victorydcesina.com \\ ${ }^{3}$ Xingzhi College, Zhejiang Normal University, Jinhua, China \\ hufeng@zjnu.cn
}

\begin{abstract}
Despite considerable efforts have been devoted to study consumer loyalty, there is a lack of knowledge concerning how online service loyalty is or can be established in a competitive e-service market, in which several major service providers coexist to compete for customers. In this study, we attempt to explore the industry environment of Chinese social networking service (SNS), and examine the association between consumer satisfaction and switching costs in building service loyalty. From a resource-based view, unique service resources of SNS (critical mass and supplemental entertainment) are examined regarding their potentials in enhancing consumer satisfaction, habit and switching costs. The results show that habit and the interaction effect of satisfaction and switching cost are the key determinants of SNS loyalty. Critical mass and supplemental entertainment directly or indirectly affect habit and switching costs. This study attempts to bring the thought of competitive environment into e-service loyalty research while new insights for e-service loyalty building in different market environments are discussed.
\end{abstract}

Keywords: Social networking, service loyalty, habit, critical mass, switching cost, satisfaction, supplemental entertainment, SNS.

\section{Introduction}

"Even though the results of customer-satisfaction surveys are an important indicator of the health of the business, relying solely on them can be fatal [17, p. 4]."

Today's enterprises are continually looking for ways to enhance customer loyalty mainly through improving consumer satisfaction. Loyal customers are vital to the long-run profitability of any business as acquiring a new customer may cost as much as five times more than retaining an extant customer [23]. Consequently, it is not astonishing that building customer loyalty has been acknowledged as an integral part of doing business or the 'business back-bone' [14]. In currently increasingly service-

* Corresponding author.

H. Li et al. (Eds.): I3E 2014, IFIP AICT 445, pp. 79-90, 2014.

(C) IFIP International Federation for Information Processing 2014 
based economies [11], a growing interest has been dedicated to consumer loyalty building in an e-service or Internet environment.

A considerable number of studies on e-service loyalty (e-loyalty) have been conducted in recent years, most of which suggest that consumer loyalty, as a result of consumer satisfaction, is mainly gained through improving service quality. However, previous works have claimed that the effect of satisfaction on consumer loyalty varies greatly under the condition of different levels of switching costs in, i.e. various human-mediated physical service environments [18]. There is lack of relevant knowledge and researches in the contexts of e-service community. For instance, little knowledge is available concerning how e-loyalty is altered along with the evolution of business environment, in particular when the market becomes highly competitive with several competent service providers.

In addition, website design tools are under rapid development with the advances in information and communication technology (ICT), which has made it an easy task to develop or even 'duplicate' new website functionality or applications. As a result, it becomes more difficulty today for e-service providers to gain advantages in competition simply relying on a well-working service website, in particular when competitors start to 'learn' fast from each other. In an Internet environment, where competition may be only one click away [36], a rising concern is how to make their service distinguishing, i.e. through offering unique resources.

From a resource-based view, the study sought to investigate the interdependencies between unique e-service resource, habit, consumer satisfaction and switching costs in building consumer loyalty in a highly competitive service market - the social networking service (SNS) market in China. In this market, several competent SNS suppliers coexist to compete for consumers while offering similar web functionalities and presentation. Two unique resources underlying SNS-critical mass and supplemental entertainment, are discussed and examined regarding their roles in facilitating consumer habit, satisfaction and switching costs.

\section{Theoretical Background}

\subsection{Determinants of E-Loyalty: Service Quality and Satisfaction}

Customer loyalty has long been a hot research topic for both business and marketing. The last several decades have witnessed a paradigm shift from tangible products (i.e. brand loyalty) to human-mediated service (i.e. hotel and restaurant) and to recent computer-mediated service (or e-service) in this field, along with the advance of information technologies. According to various researches on customer loyalty, the framework of service quality-satisfaction-consumer loyalty or its extension has been widely employed in tangible products and human-mediated service contexts [6, 14]. Apparently, e-service loyalty has been mainly assumed to be a result of satisfaction, which is enhanced by a diversity of service quality.

Despite the widespread emphasis of satisfaction on e-loyalty establishment, recent research on switching behavior of e-service seems to offer a different picture. "New IT choices are only a click away on the Internet, and switching to a competing IT is almost as easy as downloading and installing it, or completing an online registration 
form to sign up for a different service" [5]. As a result, it becomes increasingly easier for online consumers to switch to other service providers who offer similar services[36]. In certain e-service market (i.e. online gaming industry), it seems to be especially difficult to build customer loyalty, while satisfied consumers may easily switch to other service providers [c.f. 25]. A recent study on massively multiplayer online role playing games (MMORPGs) found that high attractiveness of alternatives and low switching cost significantly motivate game players' switching intention [16]. Steenkamp and Baumgartner (1992) noted that switching behaviors may occur among satisfied customers due to their attempt to try something novel or different for fun or thrill [30]. Customers may be satisfied with their choices, but may still switch to alternatives owing to a desire for novelty or complexity in brand consumption or curiosity, or getting bored, fed up, on repetitively doing the same thing $[12,15,28]$. Balabanis et al. (2006) found that satisfaction is a significant predictor of e-loyalty only for those consumers who have a low level of satisfaction [3]. Bhattacherjee et al. (2012) reported that satisfaction with prior e-service provider only has a small and marginal negative influence on switching behavior [5].

\subsection{Business Environment and Consumer Categories}

The inconsistence in previous research findings between e-service loyalty and switching behavior may be interpreted from the perspective of business environment. By an investigation of a diversity of tangible products and physical services, a study of Jones and Sasser (1995) noted that a consumer's perceived satisfaction affects loyalty in a different way in different industry settings [18], as shown in Figure 1. An consumer may be loyal to a specific service with a low degree of satisfaction, but also betray a service provider in spite of a high degree of satisfaction [c.f. 17]. As a result, based on the interaction patterns between satisfaction and loyalty, Jones \& Sasser (1995, p. 11) suggested that "consumers behave in one of four basic ways: as loyalists, as defectors, as mercenaries, or as hostages", as shown in Table 1.

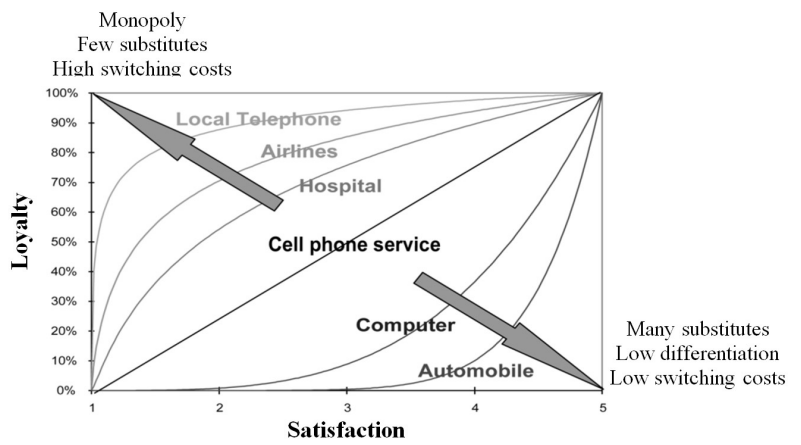

Fig. 1. How the competitive environment affects the satisfaction-loyalty relationship (Source: adapted from [18, 22]) 
In a highly competitive and low differentiation market, consumers are more likely to be low loyal whilst having a high level of satisfaction, therefore being mercenary. Based on the work of Jones \& Sasser (1995), more mercenary consumers will appear in e-service markets as a result of increased competition and decreased differentiation [18]. For instance, Hsiao \& Yang (2011) noted that a critical issue in online retailing research is how to identify, attract, and retain customers since online shoppers are typically regarded as less loyal [17].

Table 1. Individual customer satisfaction, loyalty, and behavior (adapted from: Jones \& Sasser, 1995)

\begin{tabular}{|l|c|c|l|}
\hline & Satisfaction & Loyalty & \multicolumn{1}{c|}{ Behavior } \\
\hline Loyalist & High & High & Staying and supportive \\
\hline Defector & Low to medium & Low to medium & Leaving or having left and unhayppy \\
\hline Mercenary & High & Low to medium & Coming and going; low commitment \\
\hline Hostage & Low to medium & High & unable to switch; trapped \\
\hline
\end{tabular}

\subsection{A Resource-based View of E-Loyal Building}

From a resource-based view, a firm's performance is founded on a collection of physical and intangible resources (assets or capabilities) that enable it to compete with other firms [34]. Different industry may feature different sorts of resources. For instance, Barney (1991, p. 101) defined resources as "all assets, capabilities, organizational processes, firm attributes, information, knowledge, etc. controlled by a firm that enable the firm to conceive of and implement strategies that improve is efficiency and effectiveness"[4]. A good firm resource should be valuable, heterogeneous, immobile, and non-substitutable, and therefore lead to sustainable competitive advantage of the firm [24]. Previous works suggest that unique service resources may contribute to important dimensions of service quality, which further enhances consumer satisfaction and therefore loyalty. These resources may be external in nature, such as good location and transportation convenience of a hotel[20,32], or internal, such as personal friendship to customer, routes availability in online flight booking service [c.f. 7]. Since the method of a resource-based view has been widely applied to investigate physical brand and tangible service, this method should be applicable to e-service contexts. In other words, e-service providers should seek to explore and develop unique service resources in order to make their services distinguishing and to achieve competitive advantages. It is noted that, pertaining to different natures of e-service categories, previous studies have identified a number of unique service characteristics that affect service acceptance [19, 29], many of which can be regarded as a sort of e-service resources.

\subsection{Highly Competitive E-Service Market: New Challenges to Loyalty Research}

Of various Internet services in China, Tencent QQ (QQ), a free instant messaging computer program, is obviously one of the most successful applications. In September 2011, the active QQ user accounts for QQ IM amounted to 711.7 million while its peak concurrent users reached 145.4 million (Tencent, 2011). Note the fact that there 
were 513 million Internet users in China in $2011[10]^{1}$. Whilst QQ is initially developed for instant communication, the company seeks to integrate a diversity of value-added services to the QQ and develops it to be a customized "one stop for all" application. An increasing amount of Internet services have been integrated to the simple interface of QQ (like MSN). As a result, through simply clicking on icons in its interface, QQ users can access a diversity of various Internet services, such as email, online music, games, web TV, online shopping, Internet disk, SNS and many others. One stop for all application strategy offers QQ unique advantages compared to other service providers, as there is no more need for users to make additional registration and to remember additional account and password information towards a new service supplier. In addition, friends list of instant messaging service can be easily migrated to SNS, which saves QQ users' lots of efforts to establish a new network for SNS. Also as an integrated function, new updates from SNS (i.e. comments, new posts) will be presented to users through the simple interface of QQ. Considering various inherent advantages of SNS at QQ, it seems that it should be easy to convert loyal QQ instant messaging users to be loyal SNS users alike.

However, as one of the earliest SNS providers, market share of QQ's SNS (or Qzone) has been eaten away by more recent entrants to the industry. Many users shift to other SNS providers despite their loyalty to QQ IM and give up various benefits offered. They are willing to use other SNS services, even if they have to spend additional efforts to open the browser, to fill in account and password information and to search people for reconstructing their new network. Currently, there are 190 million active SNS users for Qzone, 96 million for Renren.com and 40.1 million for Kaixin001.com [31]. The SNS market in China is dominated by several suppliers that provide similar services. After several years of development and competition, SNS suppliers share considerable similarities in their website presentation and functionalities despite limited differences. In this regard, it would be interesting to scrutinize what are unique service features (or resources) to distinguish a SNS supplier from a user's perspective.

\section{Research Framework and Hypotheses Development}

To evaluate SNS service loyalty, a research framework is proposed. Five hypothesized determinants of loyalty are included, which are habit, satisfaction, switching costs, supplementary entertainments and critical mass.

Satisfaction can be defined as a personal's feeling of pleasure or disappointment resulting from comparing a service's perceived performance (or outcome) in relation to his or her expectations [21]. Previous literature provides unambiguous supports for the positive influence of satisfaction on customer loyalty. Consistent with previous studies, it is expected that the same influence works in SNS alike. Hence, we hypothesized:

H1: Satisfaction positively relates to consumer loyalty.

In service loyalty research, switching costs measure a consumer's perceived difficulty in switching to a new service supplier, which represents anything that

\footnotetext{
${ }^{1}$ Some Internet user may actively use two or more QQ accounts.
} 
makes it more difficult or costly for consumer to change providers [8]. Switching costs are not only economic in nature, but also can be emotional or psychological, such as interpersonal relationships and special treatment $[8,36]$. Many studies suggest switching costs exert a direct influence on service loyalty, arguing that if consumers encounter a high switching costs, they are more likely to stay with the service supplier $[2,8,35,36]$. Therefore, we hypothesized:

H2: Switching costs positively relate to consumer loyalty.

Jones and Sasser (1995) pointed out that the degree of switching costs differ a lot in different service sectors, which may alter the effects of satisfaction on service loyalty [18]. Balabanis et al. (2006) classified satisfaction to be high, moderate and low levels, and hypothesized that influence of perceived switching barriers on e-store loyalty is greater when satisfaction is low [3]. Yang and Peterson (2004) divided e-service users to be two subgroups of unsatisfied and satisfied users, and reported a significant interaction effects of switching costs and satisfaction on customer loyalty in satisfied user group [36]. Lee et al. (2001) studied mobile phone service loyalty and reported a positive interaction effect of switching costs and satisfaction on loyalty [22]. Jones \& Sasser (1995) suggested that, in a high competitive and low differentiation market, people become mercenary and have a low commitment. Therefore, it is important to build switching costs to retain customers [18]. Therefore, we hypothesized:

$\mathrm{H} 3$ : Interaction effects between satisfaction and switching costs positively relate to consumer loyalty.

Habit can be defined as the "learned sequences of acts that become automatic responses to specific situations, which may be functional in obtaining certain goals or end states" [33]. The concept and function of habit has been broadly investigated across a diversity of disciplines, like social psychology, health sciences, marketing/consumer behavior, IT user behavior and organizational behavior [9]. Frequently performed behaviors tend to become habitual and hence automatic over time [26]. Habitual behavior is difficult to resist and substantial conscious efforts are needed for individuals in order to alter their habits. Therefore, altering habit in order to switch to another service provide may exert to be a sort of switching cost. Furthermore, consumers may express their loyalty to an e-service provider simply as a result of habit. In other words, they are 'lazy' to make a change or feel difficult to change their habit of being 'loyal' to existent service provider. Accordingly, the following hypothesis is made:

H4a: Habit positively relates to switching cost.

H4b: Habit positively relates to loyalty.

From a resource-based view, unique resources or capability of a company means the unique service attributes help to discriminate a service provider from others to enhance competitiveness. Unique resources can be internal, accumulated with times and co-created with customers, i.e. hotel stuff's personal relationship or friendship to customers [7]. Concerning SNS, supplementary entertainments can be regarded as a sort of internal resources. Specifically, it refers to the entertainments/games offered by SNS; these entertainments/games are not specifically designed for information sharing or communication purpose, but to offer entertainment that a user can play to compete with peers even when peers are offline. The strategy of providing 
supplementary entertainments has been dominantly applied by today's SNS providers. Taking 'Happy Farm' social game for instance, it is one of the most popular social networking games in China. At the height of its popularity, there were 23 million daily active users who $\log$ in to the game at least every 24 hours $^{2}$. As a value-added element for SNS, a provision of supplementary entertainments potentially brings about more pleasure to SNS user, making consumers more likely to feel satisfied. Therefore, it is proposed:

H5a: Supplementary entertainments positively relate to satisfaction.

Furthermore, frequently interacting with the supplementary entertainments may contribute to a motivator of forming habitual behavior to access SNS. In other words, supplementary entertainments may contribute to the establishment of habitual use of SNS. Therefore, it is proposed:

H5b: Supplementary entertainments positively relate to habit.

External resources refer to the service attributes, which are not generated by the service provider, but contribute to be an important aspect of service, such as the transportation convenience to a hotel customer. Concerning SNS, critical mass is proposed to affect consumer service experience as a kind of external resources. It refers to an important segment of population that chooses to take part in the SNS $[1,25]$. Critical mass makes major contributions to the collective action as well as to later subscribers $[1,25]$. A new network by its nature requires a group of subscribers if it is to startup; the network becomes mature to move beyond that point in its development where a critical mass has initially assembled [1]. As a result, new subscribers to a mature SNS can join one after another instead of as a group [1]. For instance, a user subscribes to a SNS, for that his/her friends are there already; as a result, s/he subscribes to the SNS by join the already existed circle of his/her friends with no need to establish a new network by his/her own efforts. Hence, users may decide to be loyal to a specific SNS so that they can connect to the people who are important to them, such as friends and relatives. The possibility to communicate with important others inside the network should be an important purpose of using SNS, which enhance their satisfaction to the service. Furthermore, when users have lots of friends using the SNS, it is more likely for them to communicate with their friends via SNS and therefore make the SNS use to be habitual. Therefore, we proposed:

H6a: Critical mass positively relates to habit.

H6b: Critical mass positively relates to switching costs.

\section{$4 \quad$ Research Methodology}

\subsection{Survey and Questionnaires}

As there are many SNS operators in China, Qzone, the biggest social networking service provider in China, is selected as the subject for survey. A five-point Likertscale ranging from disagree (1) to agree (5) was used to measure each item of the model. The questionnaire survey was conducted online. We provided a hyperlink to

\footnotetext{
${ }^{2}$ See. http://game.dayoo.com/200911/20/68602_100420554.htm
} 
the survey web page and posted it to different forums, while the respondents would be directed to the online version of the questionnaire by clicking on the hyperlink. Finally, 228 complete samples were collected, seven of which have no prior use experience and were therefore omitted from the analysis. The final samples consist of 99 males $(44.8 \%)$ and 122 females $(55.2 \%)$. Most respondents are under 25 years old $(n=175)$ and have used computers for over 3 years $(n=194) .73 .3$ percent $(n=162)$ of participants have an experience of using Qzone for over 3 years while 44 respondents $(19.9 \%)$ has $1-3$ years use experience. Only 15 participants $(6.8 \%)$ have a use experience of less than 1 year.

\subsection{Reliability and Validity of Measurement}

Partial Least Squares (PLS) path modeling technologies were utilized to analyze the data using SmartPLS 2.0. Convergent validity indicates the degrees to which the items of a scale that are assumed to be theoretically associated are also related in reality. As shown in Table 3 and 4, all values of Cronbach's alpha, factor loadings and CR are over their thresholds of 0.5 and 0.7 respectively.

Discriminant validity refers to whether the items of a scale reflect the construct in question or reflect another related construct. It can be verified if the variances of the average variance extracted for each construct are higher than any squared correlation between this construct and any other construct, the discriminant validity is supported [13].

Table 2. Reliability and convergent validity statistics

\begin{tabular}{llll}
\hline Construct & $\alpha$ & CR & Minim. factor loading \\
\hline Satisfaction & 0.941 & 0.957 & 0.907 \\
Switching costs & 0.871 & 0.912 & 0.817 \\
Supplementary entertainments & 0.820 & 0.917 & 0.917 \\
Critical mass & 0.883 & 0.944 & 0.944 \\
Habit & 0.969 & 0.980 & 0.961 \\
Loyalty & 0.923 & 0.951 & 0.904 \\
\hline
\end{tabular}

Table 3. Discriminant validity

(The bold diagonals are the AVEs of the individual constructs; off diagonal values are the squared correlations between constructs)

\begin{tabular}{llllllll}
\hline Construct & Mean & $\mathrm{Sa}$ & $\mathrm{Sc}$ & $\mathrm{Se}$ & $\mathrm{Cm}$ & $\mathrm{Ha}$ & Lo \\
\hline Satisfaction (Sa) & 3.26 & 0.850 & & & & & \\
Switching costs (Sc) & 2.60 & 0.149 & 0.722 & & & & \\
Supplementary entertainments (Se) & 3.19 & 0.247 & 0.199 & 0.847 & & & \\
Critical mass (Cm) & 3.45 & 0.168 & 0.127 & 0.098 & 0.895 & & \\
Habit (Ha) & 2.96 & 0.565 & 0.596 & 0.383 & 0.561 & 0.942 & \\
Loyalty (Lo) & 2.45 & 0.304 & 0.544 & 0.165 & 0.202 & 0.750 & 0.868 \\
\hline
\end{tabular}




\subsection{Data Analysis and Model Assessment}

Figure 2 depicts the results of model testing. As shown in the Figure, both satisfaction and switching cost alone have no significant effect on loyalty. Instead, the interaction effect of satisfaction and switching cost has significant influence on loyalty. Furthermore, habit exerts a substantial and strong influence on both loyalty and switching cost. Critical mass exerts a significant influence on habit, but not on switching cost. Supplementary entertainments significantly affect both habit and satisfaction. Critical mass and supplemental materials have indirect influences on switching cost, which are mediated by habit. The model interprets 35.6 percent of variance of switching cost, 36.3 percent of habit, 24.9 percent of satisfaction and 72 percent of loyalty.

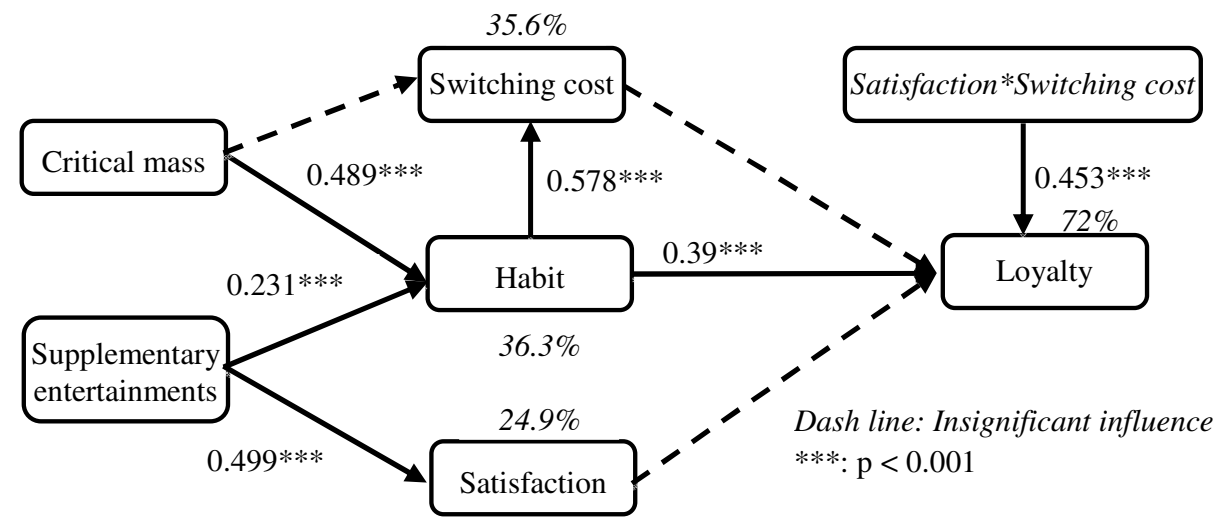

Fig. 2. Results

\section{$5 \quad$ Discussion and Implications}

Figure 2 shows that, in this competitive SNS market, consumers tend to be mercenary, whereas a high satisfaction of SNS users doesn't guarantee a high loyalty toward service provider. In addition, satisfaction alone cannot bring about loyalty as well. Consistent with the work of Jones \& Sasser (1995), we propose that consumer satisfaction may not be such a reliable indicator of loyalty for e-service market, in particular when the market becomes more competitive with less differentiation. Consumers behave differently in response to different industry environments. In other words, the change of industry environment itself may effectively alter the way that consumers choose to be loyal to a company, i.e. mercenary versus hostage consumers. The results show a strong interaction effect between satisfaction and switching costs on SNS loyalty. In other words, it is really difficult to establish user loyalty in SNS: only when consumers are satisfied with service provider and are feeling difficulty to switch, they become loyal. This also suggests that satisfaction is a necessary condition of service loyalty. Satisfied consumers can be either loyal or not loyal to service provider based on the level of switching cost, but dissatisfied consumers definitely 
exert no loyalty. Habit has in particular strong and direct influence on SNS loyalty. This implies that, in Chinese SNS market, consumer loyalty is more like a sort of habitual behavior. In other words, there is a high risk of consumer switch, if they are strongly motivated to change their habit, e.g. by their peers (critical mass).

Furthermore, critical mass and supplementary entertainments are found to be important resources that significantly enhance consumer habit, satisfaction and switching costs on SNS. This indicates that, if a SNS maintained an individual's network by hosting the people who are important to him/her, the user is less likely to switch because it will cause a loss of connections to those important people. Also the time and efforts spent on supplementary entertainment, such as social networking games, appears to be valuable to the consumers. They feel more satisfied if they engage in supplementary entertainment, in particular when they play together with their friends.

\section{Conclusion}

In this study, we sought to introduce a resource-based view into the field of e-service research, and to interpret how unique service resources help establish both satisfaction and switching costs. Whilst the resource-based view has been dominantly utilized to study the competitiveness of different companies and organization, this approach is novel to the field of e-service research. Based on the research findings of this study, we argued that it is important to recruit and develop unique service capabilities in order to make the e-service distinguishing and to achieve competitiveness.

While previous works have examined the influence of industry settings in affecting satisfaction-loyalty relationship mainly concerning human-mediated service industries [18], our study investigates the loyalty building in a competitive e-service market with several competent suppliers. The results of this study illustrate that, in Chinese SNS market, despite of a high satisfaction, consumer loyalty cannot be established without a proper level of switching costs. In Internet environment, the choice of websites is already huge and increasing. Therefore, it is essential for websites to focus more on increasing switching costs than eliminating alternatives. In this regard, our results facilitates a more complete understanding on e-service loyalty building in context and seeks to raise the research interests in unique service resources for developing distinguishing e-service. Previous studies show that switching costs vary in different industry settings while the effects of satisfaction on customer retention will be deteriorated in case of high switching costs [22]. Simply considering e-service loyalty from the view of satisfaction, important information might be underexplored, in particular if industry environment and switching costs become a matter of importance. There is a dearth of relevant wisdom in the e-service field concerning some new and emerging business phenomena, such as the predicament faced by Qzone.

Acknowledgements. This research is partly supported by Wuhan University Academic Development Plan for Scholars after 1970s for the project Research on Internet User Behavior and the National Funds of Social Science (No. 14BTQ044), 
the National Planning Office of Philosophy and Social Science in China (No. 13CTQ029) and the Zhejiang Province Planning Project of Philosophy and Social Sciences (No. 11JCGL04YB).

\section{References}

1. Allen, D.: New telecommunications services: Network externalities and critical mass. Telecomm. Policy. 12(3), 257-271 (1988)

2. Amin, S.M., et al.: Factors Contributing to Customer Loyalty Towards Telecommunication Service Provider. Procedia - Soc. Behav. Sci. 40(6), 282-286 (2012)

3. Balabanis, G., et al.: Bases of e-store loyalty: Perceived switching barriers and satisfaction. J. Bus. Res. 59(2), 214-224 (2006)

4. Barney, J.: Firm resources and sustained competitive advantage. J. Manage. 17(1), 99-120 (1991)

5. Bhattacherjee, A., et al.: User switching of information technology: A theoretical synthesis and empirical test. Inf. Manag. 49(7-8), 327-333 (2012)

6. Bloemer, J., et al.: Linking perceived service quality and service loyalty: a multidimensional perspective. Eur. J. Mark. 33(11/12), 1082-1106 (1999)

7. Butcher, K., et al.: Evaluative and relational influences on service loyalty. Int. J. Serv. Ind. Manag. 12(4), 310-327 (2001)

8. Chang, Y.-H., Chen, F.-Y.: Relational benefits, switching barriers and loyalty: A study of airline customers in Taiwan. J. Air Transp. Manag. 13(2), 104-109 (2007)

9. Chou, C., et al.: Understanding Mobile Apps Continuance Usage Behavior and Habit: An Expectance-Confirmation Theory. In: PACIS 2013 Proceedings. p. Paper 132 (2013)

10. CNNIC: The 29th Statistical Report on Internet Development in China (2012)

11. Dai, H., et al.: Antecedents of online service quality, commitment and loyalty. J. Comput. Inf. Syst. Winter, 1-11 (2011)

12. Fiske, D.W., Maddi, S.R.: Functions of varied experience. Dorsey Press, Homewood (1961)

13. Fornell, C., Larcker, D.: Evaluating structural equation models with unobservable variables and measurement error. J. Mark. Res. 18(1), 39-50 (1981)

14. Gremler, D.D., Brown, S.W.: Service loyalty: its nature, importance, and implications. In: Edvardsson, D.D., et al. (eds.) QUIS V: Advancing Service Quality: A Global Perspective, NY, New York, pp. 171-181 (1996)

15. Herrnstein, R.J., Prelec, D.: A theory of addiction. In: Loewenstein, G. and Elster, J. (eds.) Choice Over Time. Russell Sage Press, New York (1992)

16. Hou, A.C.Y., et al.: Migrating to a new virtual world: Exploring MMORPG switching through human migration theory. Comput. Human Behav. 27(5), 1892-1903 (2011)

17. Hsiao, C.H., Yang, C.: The intellectual development of the technology acceptance model: A co-citation analysis. Int. J. Inf. Manage. 31(2), 128-136 (2011)

18. Jones, T., Sasser, W.: Why satisfied customers defect. Harv. Bus. Rev. 76(3) (1995)

19. Kim, J., et al.: The role of etail quality, e-satisfaction and e-trust in online loyalty development process. J. Retail. Consum. Serv. 16(4), 239-247 (2009)

20. Knutson, B.: Frequent travelers: making them happy and bringing them back. Cornell Hotel Restaur. Adm. 29(1), 82-87 (1988)

21. Kotler, P.: Marketing management. Prentice-Hall, Englewood Cliffs (2003)

22. Lee, J., et al.: The impact of switching costs on the customer satisfaction-loyalty link: Mobile phone service in France. J. Serv. Mark. 15(1), 35-48 (2001) 
23. Madhavan Parthasarathy, A.B.: Understanding post-adoption behavior in the context of online services. Inf. Syst. Res. 9(4), 362-379 (1998)

24. Mata, F., et al.: Information technology and sustained competitive advantage: a resourcebased analysis. MIS Q. 19(4), 487-505 (1995)

25. Oliver, P., et al.: A theory of the critical mass. Am. J. Sociol. 91(3), 522 (1985)

26. Ouellette, J.A., Wood, W.: Habit and intention in everyday life: The multiple processes by which past behavior predicts future behavior. Psychol. Bull. 124(1), 54-74 (1998)

27. Playnomics: Playnomics Quarterly US Player Engagement Study Q3 2012, http: / /ww1 .prweb.com/prfiles/2012/10/18/10028632/Q3reportv4. pdf

28. Raju, P.S.: Optimum Stimulation Level: Its Relationship to Personality, Demographics, and Exploratory Behavior. J. Consum. Res. 7(3), 272-282 (1980)

29. Srinivasan, S.S., et al.: Customer loyalty in e-commerce: an exploration of its antecedents and consequences. J. Retail. 78(1), 41-50 (2002)

30. Steenkamp, J., Baumgartner, H.: The role of optimum stimulation level in exploratory consumer behavior. J. Consum. Res. 19(3), 434-448 (1992)

31. TechRice: Recruiting via China's Social Networks (2012)

32. Tsaur, S.-H., et al.: Determinants of guest loyalty to international tourist hotels-a neural network approach. Tour. Manag. 23(4), 397-405 (2002)

33. Verplanken, B., et al.: Habit, information acquisition, and the process of making travel mode choices. Eur. J. Soc. Psychol. 27, 539-561 (1997)

34. Vinekar, V.: Towards a Unified Model of Information Technology Business Value. In: AMCIS 2006 Proc. (2006)

35. Wang, Y.-S., et al.: The relationship of service failure severity, service recovery justice and perceived switching costs with customer loyalty in the context of e-tailing. Int. J. Inf. Manage. 31(4), 350-359 (2011)

36. Yang, Z., Peterson, R.T.: Customer perceived value, satisfaction, and loyalty: The role of switching costs. Psychol. Mark. 21(10), 799-822 (2004) 